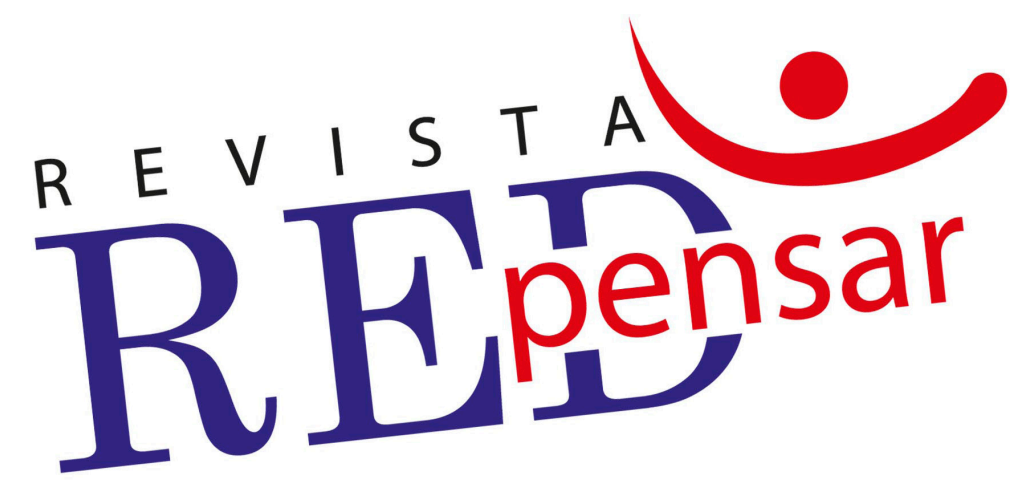

\title{
Perspectiva de Aprendizaje Ecológico
}

Ecological Learning Perspectives

Melissa González Castro

Revista REDpensar, volumen 7, número 2, Julio-Diciembre 2018 ISSNe: 2215-5384

REDpensando en la Investigación 1 Recibido: 20 de octubre de 2018 Aceptado: 29 de noviembre de 2018

DOI: 10.31906/redpensar.v7i2.167

\section{(ㄷ)(1)(2)}




\title{
Perspectivas de Aprendizajes Ecológicos
}

\author{
Ecological Learning Perspectives
}

Melissa González. Castro ${ }^{1}$

\section{Resumen}

Lo planteado en este artículo tiene que ver con formas de potenciar en el ser humano la capacidad de renovarse como una totalidad fluyente y sin fronteras, en el ámbito educativo. De esta manera, aprende desde sus propios cambios estructurales, influenciado por procesos externos, sobre la construcción del pensamiento, el conocimiento, las habilidades y la cultura. Es así, que en la primera parte se hace una analogía entre el modelo biométrico fragmentario de la enfermedad y el modelo educativo que se desarrolla a nivel Latinoamericano, en oposición, a los escenarios que impulsan la emergencia de aprendizaje como los que promueven Gutiérrez y Prieto (2002) educar en y para la incertidumbre, el gozo en la vida, la significación, la expresión, la convivencia y el apropiarse de la historia y de la cultura. Estos escenarios, propios del paradigma emergente sitúan al ser humano como proyecto de sí mismo, y para esto, se propone la mediación pedagógica desde la persona que aprende a través de sus sentidos.

Palabras Clave: INTERDISCIPLINARIEDAD, ECOLOGÍA, EDUCACIÓN, APRENDIZAJE, CREATIVIDAD.

\section{Abstract}

The issues raised in this article is about ways to enhance the human capacity to renew itself as a flowing whole without frontiers in education. In this way, learn from their own structural changes, influenced by external processes on the construction of thought, knowledge, skills and culture. Thus, in the first part an analogy between the biometric model Fragmentary disease and the educational model developed in Latin America, as opposed to scenarios that drive the emergence of learning as those promoting Gutierrez and Prieto done (2002) educate in and for uncertainty, joy in life, meaning, expression, coexistence and appropriation of history and culture. These scenarios, typical of the emerging paradigm situate the human being as the project itself, and for this, is proposed the pedagogical mediation from the person who learns through from his senses.

Keywords: INTERDISCIPLINARITY, ECOLOGY, EDUCATION, LEARNING, CREATIVITY.

1. Máter en Ciencias de la Educación con Énfasis en la Enseñanza del Inglés. Labora actualmente para el Ministerio de Educación Pública de Costa Rica (MEP), Departamento de Inglés, y en los Programas de Idiomas de Extensión Comunitaria de la Universidad Técnica Nacional de Costa Rica (UTN). Correo electrónico: meli goca@yahoo.com 


\section{Introducción}

Desde la visión mecanicista del mundo en el siglo XVII, Descartes plantea la metáfora de que el ser humano es igual a una máquina que funciona sincrónicamente como un engranaje donde cada una de sus partes tiene una función importante. Evidentemente, este planteamiento ha calado en la civilización occidental con el pasar de los años. Y, tiene aún relevancia en pleno siglo XXI, donde el modelo biométrico de la enfermedad y la educación y muchos otros campos reflejan el mismo enfoque reduccionista de la realidad. Desde esta perspectiva, el ser humano ha sido pensado y adoctrinado de forma fragmentada. La distinción entre cuerpo y mente ha sido tan fuerte, que el ser humano es educado para satisfacer los deseos de su cerebro primitivo, es decir, los deseos que hagan a su cuerpo sentirse satisfecho, como abrigo y comida. Por lo que, desde el vínculo más pequeño de la sociedad, o sea la familia, el individuo es enseñando a estudiar y trabajar para aquello que le ostente de abrigo y comida, olvidando que la persona no es un ser dividido solamente en cuerpo y mente como se creía en siglos atrás.

Las ideas actuales de la física moderna, nos enseña sobre el nuevo concepto de la teoría general de sistemas, Capra (1992) afirma

Que el ser humano ya no puede concebirse como una máquina formada por una gran cantidad de objetos, sino que ha de concebirse como una unidad indivisible y dinámica cuyos elementos están estrechamente vinculados y pueden comprenderse sólo como modelos de un proceso cósmico. (p.40)

Mi deseo es plantear formas de potenciar en el ser humano su capacidad de renovarse como una totalidad fluyente y sin fronteras en el ámbito educativo. Y con este planteamiento, el objetivo principal de este artículo es hacer una analogía entre el modelo biométrico fragmentario y el modelo educativo que se desarrolla en nuestra nación y en muchas otras en la época de nuestros días, en oposición al paradigma emergente de educación ecológica.

\section{Primer Momento: Modelo Biométrico Fragmentario en la Educación} Actual

En un primer acercamiento, quiero referirme a cómo se ha dividido el sistema educativo en dos grandes grupos de poder, el público y el privado. Por un lado, el sistema educativo público que se encuentra cobijado y patrocinado por un sistema de gobierno patriarcal, bajo una economía de mercados neoliberal capitalista global. En contraste, el sistema educativo privatizado obedece a un grupo más reducido de esta economía, la élite más adinerada que puede costear una educación más sofisticada, en otras palabras, una educación que obedece más al modelo norteamericano. En resumen, ambos grupos visualizan la educación como una gran empresa en el sistema materialista moderno en donde, según Capra (1992) "el nivel de vida se mide de acuerdo con la cantidad de consumo anual, y que por consiguiente trata de alcanzar el consumo máximo junto con un modelo óptimo de producción" (p.101). Dicho en otras palabras, esta gran empresa educativa debe llegar a modelos óptimos de producción de mano de obra barata que pueda ser pagada a un bajo precio dentro del país, pero que, a su vez pueda ser exportada de una forma económica a otros contextos y cuyos insumos puedan utilizarse cíclicamente como materia prima para masificar su producción.

Este modelo de producción en masas es promovido y potencializado por los medios de comunicación que venden en grandes dosis la visión ya empaquetada del discurso del gobierno. Este discurso alienador hegemónico va en detrimento de las clases más pobres ya que expone la crisis económica en la que se 
encuentra el país y que supuestamente sólo puede ser solucionada si las clases trabajadoras pagan de manera solidaria los impuestos que ayudarán a disminuir los niveles de inflación. Esto incluye, sin duda alguna, reducir el presupuesto nacional en educación e incrementarlo en otros campos de producción de insumos masificados e inmediatos.

Por supuesto, este discurso de globalización capitalista neoliberal que viene desde el sistema de dominio monetario es transmitido, de forma alienadora, hacia todas las esferas educativas formales para que se siga cultivando y transmitiendo de generación en generación. Una vez más el ser humano globalizado se concibe, fuera de la complejidad, como una fragmentación de sus partes y no como una totalidad. Siendo de esta manera alienado, marginalizado y aislado en reducción total de sus capacidades y autonomía. De esta manera se consolidan intereses económicos, sociopolíticos y geográficos de poder, que siguen perpetuando su discurso sobre reformas educativas, las cuales no son más que reformas económicas.

Tradicionalmente, la práctica de la enseñanza-aprendizaje popular había sido una tarea asignada a las mujeres, dado que sus hijos aprendían dentro de la familia. Anteriormente, en tiempos de conquista y colonia, el conocimiento y la verdad de las cosas estaban sólo bajo el poder de los hombres. Así mismo, los y las docentes de pueblo eran respetados en tiempos de antaño como personalidades sabias que manejaban el conocimiento de las sociedades. Hoy, esta tendencia se está invirtiendo por el movimiento feminista, en que las mujeres han tomado la batuta como educadoras de nuestros tiempos. Sin embargo, la imagen docente ha sido pisoteada por el sistema educativo actual, desprovisto de valores en que el o la docente deja de ser una persona respetada para pasar a ser una persona señalada y marginalizada; además, como si fuera poco, su labor docente ha sido cargada con numerosas tareas administrativas y papeleo innecesario que hace que los profesores se sientan bajo una situación de estrés que va en detrimento de su labor.

El valioso poder de la mediación pedagógica ha sido reducido a una labor de transmisión de información. En consonancia con el discurso neoliberal, las y los docentes deben de reproducir en las aulas (casi jaulas humanas) un discurso adoctrinante que muchas veces no es compatible con la realidad del contexto educativo, discriminatorio y desigual. Las personas educadoras pierden prestigio y respeto ante sus ojos y el de los demás, al ser incapaces de realizar una labor por convicción personal y no por compromisos externos.

En relación a lo anterior, Capra (1992) indica "la falta de responsabilidad y de orgullo, junto con el motivo principal de los beneficios, han originado una situación en la que la mayoría del trabajo es hoy un gasto inútil y no tiene justificación" (p. 124). De esta manera, los cuatro pilares de la educación "aprender a conocer, aprender a hacer, aprender a vivir juntos, aprender a ser" (Delors, 1994, p.34) promovidos por la Organización de las Naciones Unidas para la Educación, la Ciencia y la Cultura [UNESCO] quedan en el papel.

El rol del docente debe de ser replanteado por la sociedad: así como los y las docentes fueron capaces de transformar el contexto socioeconómico y cultural de nuestros ancestros, los y las docentes actuales deben de repensar el aprendizaje desde una mirada compleja y un paradigma emergente, en donde el ser humano sea una unidad integral en relación directa con su entorno. Los y las profesionales en educación son entes que desde su contexto educativo y extramuros (fuera del aula) pueden despertar una nueva conciencia ecológica de la mano con un sistema de valores que lleven al ejercicio de la sana convivencia, el amor, el cuidado y el sano desarrollo común desde las acciones de cada quién.

Como si fuera poco, la ciclicidad de la enseñanza-aprendizaje hacia una nueva conciencia planetaria se ve perturbado por el crecimiento no diferenciado de la tecnología. En la sociedad de nuestros días, donde todas las tareas cotidianas han sido digitalizadas, las nuevas generaciones no pueden visualizar su día a día sin el uso de la tecnología, la cual ha sido puesta al servicio de grandes y pequeños sin discriminación alguna, obviando su impacto corto y/o largo plazo. Nuestra sociedad visualiza con desprecio las labores cotidianas y simples; aquellas labores que no precisen el uso de sofisticadas tecnologías de la información han sido relegadas para personas de bajos recursos económicos o personas no letradas. En caso contrario, en toda aquella labor doméstica, laboral o académica debe de tener cabida el uso de tecnologías. En el peor de los casos, la educación de los niños, las niñas y las personas jóvenes se ha relegado a las máquinas y las aulas virtuales

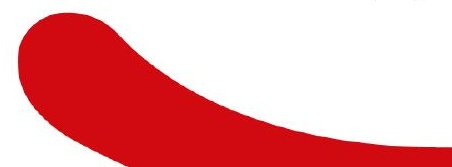


donde estos jóvenes pierden todo contacto con su entorno social y su conciencia divina.

El rol docente ha sido lentamente sustituido por módulos y videos que dicen ser educativos y reproducidos por las élites económicas moldeando al futuro ciudadano. La persona individualizada desde la perspectiva de ciudadano ya no es una persona con ideales y valores definidos, sino, una persona desprovista de arraigo a su tierra, a su familia y a sus orígenes. Este tipo de ciudadano, según el sistema económico predominante, puede ser vendido a un bajo precio a compañías nacionales y extranjeras, puede ser negociado a las grandes multinacionales, puede ser despatriado, puede ser a-culturizado sin que este proceso sea si quiera percibido. Se lleva a cabo desde edades tempranas y es reforzado por medio de las redes sociales y los medios de comunicación en masas.

Para Harari (2018) los países en vías de desarrollo que carecían de recursos naturales progresaron en el plano económico sobre todo vendiendo el trabajo barato de sus obreros no cualificados. Al respecto señala,

Hoy en día, millones de bangladesíes se ganan la vida fabricando camisas y vendiéndolas a clientes en Estados Unidos, mientras que en Bangalore lo hacen en los servicios telefónicos de atención al cliente que tramitan las quejas de los clientes norteamericanos. (p.62)

De acuerdo con Grosfoguel (2011, p. 40) "puesto que nuestra crisis actual es producto y reflejo del estadio de evolución de la conciencia de la humanidad, es inconcebible una solución radical y permanente sin una transformación interior y sin un avance hacia la conciencia global". En esta misma línea de pensamiento, Huxley et.al (1994) ponen su esperanza en una solución genuina al problema creyendo que el ser humano emerge de una naturaleza libre que no depende de tener sino de ser, que es capaz de amar y transformase a través del amor, y sentir alegría, sintiéndose en armonía con la vida y con los demás.

Este ser humano no se forma de acuerdo con los preceptos de la sociedad, sino que se auto descubre a través de su creatividad y de su conciencia de ser humano cuya capacidad divina es distinguida por muchas de las civilizaciones conocidas y desconocidas. Ciertamente, este instinto creativo debe de ser impulsado al exponerse a experiencias de vida que lo hagan destruir las barreras que han sido construidas a lo largo del tiempo con relación a muchas áreas de su vida como política, educación, economía, entre otros. Esas experiencias transcendentales deben de ser dirigidas a que el ser humano pueda aprender para la vida y encontrar asombro en los pequeños detalles de su existencia.

En otras palabras, el ser humano debe de ser tan versátil como para desaprender lo aprendido durante siglos y estar dispuesto a descubrir su estado natural de gozo, que va a dar paso a una nueva civilización ecológica. Solamente el amor, la esencia, la mística, la contemplación, la iluminación o la gracia de Dios con todos nosotros y a través de todos nosotros pueden llevarnos más allá. Personalmente, espero que mi relación con mis comunidades de aprendizaje, me permitan vislumbrar algunas de estas formas de potencializar nuestra conciencia y nuestro ser y continuar difundiendo este mensaje a lo largo del tiempo. 


\section{Segundo Momento: ¿Cómo emerger en escenarios de Aprendizaje Ecológicos?}

El cerebro humano es el órgano más potente que posee el ser humano. Desde su nacimiento, el ser humano cuenta con aproximadamente cien mil millones de neuronas, por tal hecho se puede afirmar que el ser humano nace listo para pensar y aprender. Sin embargo, desde tiempos inmemoriales, las ciencias exactas, principalmente la ciencia de la economía se ha encargado de dictar a una sociedad cómo y qué debe de aprender la persona, bajo qué esquema y bajo qué paradigma. Lamentablemente, estos paradigmas positivistas de aprendizaje han sido aceptados por la población a lo largo de los siglos, fosilizando nuestro esquema de aprendizaje y a su vez convirtiéndonos en su esclavo. Una muestra de ello son las bases del Paradigma de la Administración Científica, creado por Taylor (1994, p. 136), donde se expresa que el modelo de operario ideal es "el hombre buey" mentalmente obtuso, que debe ser adiestrado por un hombre más inteligente. No obstante, la literatura afirma que estas teorías pasaron a ser parte de nuestros antepasados, la realidad inmediata nos señala que aun en los ambientes educativos de hoy en día se promueve este prototipo de "hombre buey", que supone un ser que debe hacer lo que se les ordena; les asigna un papel pasivo, sin la posibilidad de hacer aportes con sus ideas y mucho menos, participar en las decisiones de su vida, de su aprendizaje; así se ilustra cómo todo el paradigma mecanicista fue adoptado y apropiado también por las ciencias sociales en los ambientes laborales, educativos y familiares. Sin embargo, ni el cerebro es un ordenador, ni la mente está completa sin el cuerpo. El cuerpo es lo que hace que el mundo nos importe.

El interés de lo que hacemos nace del interés corporal, necesitamos un nuevo encuentro con las humanidades y las ciencias sociales; las ciencias de la cognición precisan buscar el ser humano como proyecto de sí mismo, y para esto, se debe de concentrar en la educación de una persona que aprenda a través de los sentidos como lo plantea Varela, Thompson y Rosch (1997, p.174) en los años 90's bajo el concepto de la "enacción”.

Enseguida, exploramos algunos de los escenarios de aprendizaje que impulsan la emergencia de aprendizaje, como los que promueven Gutiérrez y Prieto (2002, p.16): “educar en y para la incertidumbre, el gozo en la vida, la significación, la expresión, la convivencia y el apropiarse de la historia y de la cultura”.

La biología cuántica reconoce que el organismo aprende a partir de las relaciones que se establecen de sí mismo con el entorno y como parte del entorno (medio ambiente vivo). Las conexiones que genere este organismo con su medio ambiente nacen de la incertidumbre de aprender, qué sucede en su entorno inmediato en un lugar y tiempo determinados. Una característica de este tipo de ambiente educativo de incertidumbre es el no proveer respuestas y el interrogar como herramienta de concepción de nuevos recorridos. Este acercamiento corresponde a una forma de aprendizaje natural, suponiendo que, un bebé desde su posición de incertidumbre adquiere los primeros aprendizajes de sobrevivencia explorando el mundo, tomando riesgos, y afrontando escenarios que le provean autoaprendizaje.

En este sentido, la escuela como institución se ha encargado de proveer un tipo de enseñanza antipedagógica que intenta llenar (como un frasco vacío) a la persona de un cúmulo de contenidos conocidos como currículo oficial que obedece a una sociedad específica en tiempo y espacio. Este cúmulo de conocimientos está permeado por la cultura y las ideologías propias de un pueblo. Este tipo de pedagogía, llena de respuestas inhibe el sentido del descubrimiento que trae un niño o una niña desde su infancia².

2. En mi caso específico, durante la escuela primaria y secundaria aprendí a memorizar extractos de la historia, leer textos de literatura universal y retener sus personajes principales y escenarios épicos, pero nunca se me facilitaron escenarios donde personalmente pudiera desarrollar habilidades para la vida. Fue hasta que ingresé a una universidad humanista donde me promovieron los procesos de abstracción e imaginación apoyados a su vez de los procesos de interpretación y análisis que realmente no eran nuevas en mi sistema cognitivo, sino que estaban a la espera de emerger y ser puestos en práctica en mi vida diaria.

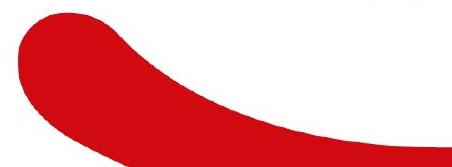


Otro de los fundamentos importantes en el planteamiento de una alternativa de aprendizaje es el aprender a gozar la vida. Desde que ingresamos a la escuela (jaula humana) se nos etiqueta como "discentes", "estudiantes", “educandos" que están aprendiendo matemáticas, ciencias, idiomas, letras, entre otros. No se nos observa como personas aprendiendo para la vida, para gozar de la vida, ni mucho menos para aprender de habilidades blandas que nos faciliten la comunicación y la interacción con otros, que podrían resumirse como el propósito primordial de la educación: fortalecer la comunicación y las relaciones para la vida. Lamentablemente, esto es lo que menos se predica entre las cuatro paredes que llamamos escuela.

Los seres humanos estamos preocupados por recibir una enseñanza que nos provea una carrera productiva y un salario competitivo, y no es hasta nuestra jubilación que nos damos cuenta que nos quedan pocos años para gozar de la vida. El gozo por la vida se cultiva adquiriendo las habilidades necesarias para resolver problemas, a esos problemas prácticos que se dan en nuestra cotidianeidad en cualquiera de los entornos en que nos desenvolvemos. Cada problema es un escenario que nos permite crecer tomando perspectivas diferentes fuera de nuestra zona de confort, donde la persona se ve perturbada y forzada a buscar soluciones que le permitan volver de nuevo a su estado de paz y estabilidad.

Este es un proceso que somos capaces de realizar desde que somos una unidad orgánica viviente con una capacidad autopoiética ${ }^{3}$, que nos permite crear conscientemente experiencias de aprendizaje. Para adquirir esta conciencia las personas deben estar atentas a su corporeidad aprendiente. La vida mental esta corporeizada. El autoconocimiento de una persona está determinado por su relación con el cuerpo, y todo el entramado del organismo y medio ambiente se auto organiza para gozar de la vida, aprendiendo a vivir y vivir para aprender.

Una vez que nos reconocemos como organismos vivos y autopoiéticos, podemos educarnos para la significación. En el entendido de cómo dar sentido a la vida, de cómo dar sentido a lo que hacemos, de incorporar el sentido de cultura y de mundo a cada persona. En otras palabras, cada organismo crea su propia identidad con respecto a sí mismo y al medio que lo rodea. Esto lo hace con la ayuda de las conexiones que crea a su alrededor a partir de la ontología de la cultura que comparta con las demás personas en su entorno.

Los niños y las niñas deben tener tiempo para jugar libremente, incluso aburrirse, porque eso hace que el cerebro haga conexiones, sobre el tomar decisiones en lo que realmente le gusta hacer. Esta interiorización del aprendizaje se da de forma fluida siempre y cuando tenga un significativo impacto en la vida del individuo. Por lo que aprendemos mejor desde los escenarios y experiencias de la vida cotidiana, sobre lo que nos genera interés, motivación y/o compromiso. Esta perspectiva se puede ver ilustrada en el caso de las personas que aprenden a hacer algo nuevo por algún tipo de interés ${ }^{4}$. Los escenarios de vida en que nos desenvolvemos nos plantean experiencias de aprendizaje y somos nosotros como organismos adaptativos quienes decidimos si crecer y desarrollarnos a partir de este aprendizaje significativo.

\footnotetext{
3. La autopoiesis se refiere a la propiedad que tienen los sistemas de auto producirse y frente a la afectación producto de la interrelación con su entorno. Para profundizar, se recomienda ver Humberto Maturana y

Francisco Varela.

4. Recuerdo que hace varios años tuve la oportunidad de adquirir un carro; sin embargo, pasaron tres años hasta que me interesé por aprender a manejar. Creo que no había aprendido a manejar anteriormente, ya que, no tenía ninguna necesidad práctica, hasta que tuve una oferta laboral lejos de mi hogar que me hizo visualizar la importancia en este nuevo aprendizaje. Esto mismo experimenté cuando quería aprender inglés, estudié el idioma varios años, pero me era muy difícil expresarme y fue hasta que me apareció la oportunidad de viajar a un país de habla inglesa que me decidí por mejorar mi idioma.
} 
Por otro lado, a partir de nuestra corporeidad somos capaces de aprender y de expresarnos; educar para la expresión implica el desarrollo de individuos pensantes. Según expresa Maturana (2002, p.140) "la mente humana a través del lenguaje, es capaz de aprender cualquier cosa". Aprender un idioma presupone uno de los procesos más complejos que aprende el ser humano, cada vez que aprendemos un nuevo lenguaje, se crean en el cerebro nuevas conexiones neuronales optimizando nuestra forma de cognizar.

Desde pequeños aprendemos a comunicarnos como mecanismo de sobrevivencia, pero, al llegar a nuestra adultez ya el lenguaje no debería de ser solamente un mecanismo de sobrevivencia sino más bien un medio para publicitar nuestra personalidad y nuestros valores, para acercarnos hacia los demás. Además, se puede afirmar que adquirir un idioma es a la vez, adquirir una nueva cultura, y conocer diferentes culturas nos acerca a la otredad, es un paso para convivir con otros.

Las personas pueden expresarse de diferentes maneras a través de sus sentidos, todas las personas nos expresamos con palabras, con gestos y con movimientos. La expresión es algo que está presente en todos, necesitamos de ella para comunicarnos, socializar y relacionarnos. Comunicarse implica desarrollar cierto tipo de habilidades para la vida como la resolución de conflictos y el pensamiento crítico. A través de la comunicación, nos vinculamos con las demás personas y con esto se promueve la convivencia humana. Educar para la convivencia y la paz es una propuesta de esperanza y posibilidad para atender asuntos apremiantes que inciden en el aprendizaje y la vida de las personas. Ante la cultura de violencia que prevalece en muchos de nuestros países y globalmente, aprender a convivir es uno de los desafíos más importantes que enfrentamos en la educación, en la actualidad.

En nuestro país, la familia, la iglesia y la escuela se disputan el primer lugar para la enseñanza de los principios de convivencia que tanto hacen falta en la sociedad. Ciertamente, no podemos aprender a convivir sino con las demás personas, y las personas van a aprender sobre las reglas de convivencia en cualquiera de los campos de la vida en el que se desenvuelva. El aprender a convivir es uno de los pilares propuestos por la UNESCO (Delors, 1994), y envuelve el respetar a los Otros en proyectos conjuntos y trabajo cooperativo. Sin embargo, la estructura gaseosa volátil de nuestra sociedad pone en conflicto día a día la convivencia social.

Hoy en día, los cambios en la convivencia se precipitan debido a las redes sociales que se han convertido en los medios de convivencia por excelencia, entre la niñez y la adolescencia, quienes pasan horas y horas frente a su teléfono móvil o a su computador "relacionándose" con otros; es por esto, que emergen nuevas reglas de convivencia. Las redes sociales necesitan de mediación y acompañamiento ya que deben ser en todo momento una herramienta y no un fin (Blanco, Seco y Vargas, 2015)

Finalmente, la convivencia entre nosotros los humanos, debe ser una acción que se debería de promover en todo ámbito, desde el núcleo de la familia, los círculos de amigos, amigas, los y las docentes; de tal manera que se convierta en un hábito que origine resonancia mórfica en el cosmos a través de los siglos, y así, emerjan formas de convivencia ecológica más sanas 5

Freire (2008, p. 69) expresa que la educación es la construcción de conocimiento, sin embargo, "nadie educa a nadie, nadie se educa a sí mismo, los hombres se educan entre sí con la mediación del mundo". El aprendizaje significativo y experimental se da mediante la apropiación de la conciencia sobre las raíces de la historia y la cultura. Cuando se aprende sobre historia aprendemos de dónde venimos, de nuestras raíces y de nuestros antecesores. Cuando aprendemos de cultura creamos relaciones más sanas con los demás.

La educación por la diversidad cultural y la convivencia es un tema que ha sido vetado en muchas naciones, dado que persiguen principios religiosos, sociales y económicos que favorecen a unos pocos y dejan de lado la cimentación de una sociedad solidaria.

5. En el sentido de interrelacionalidad con las diferentes formas cósmicas que se traduce en armonía universal.

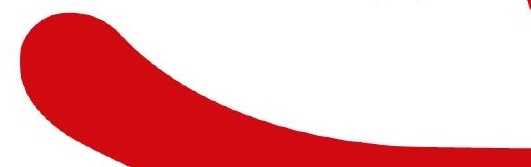


En la antigüedad, los niños y las niñas, y los y las adolescentes eran educados desde el seno familiar donde se transmitía de generación en generación a través de la narración oral la cultura y tradiciones de la familia ${ }^{6}$. Hoy en día, la educación de costumbres y tradiciones se ha delegado a otras instancias que, lejos de ser las correctas, no promulgan sobre valores, ni relaciones de sana convivencia; por ejemplo: los medios de comunicación y desinformación. Si bien es cierto, no es necesario ser gran historiador para conocer sobre historia y cultura, en las familias encontramos padres, madres, abuelos, abuelas, hermanos y hermanas con un vasto cúmulo de conocimiento que no ha sido adquirido en la escuela ni en la universidad, sino, a lo largo de las enseñanzas de la vida. Penosamente, ya la mayoría de las familias no están tan comprometidas al compartir de vivencias y experiencias intrafamiliares en el lenguajeo amoroso, sino que la mayor parte de nuestro tiempo la invertimos en el trabajo; ya no vivimos para gozarnos y amarnos, sino más bien, vivimos bajo un régimen capitalista que nos reprime en la producción laboral. A la larga, los algoritmos biométricos ideados por los imperios capitalistas aprenden a componer canciones enteras, jugando con las emociones humanas, como si fueran el teclado de un piano. Utilizando de esta manera nuestros datos biométricos que sólo nosotros, en todo el universo, apreciaríamos como creación Divina.

\section{Conclusiones}

Así es el proceso de vida de los seres aprendientes como lo expresa Assmann (2002)

Las ciencias de la vida están demostrando que la vida es básicamente una persistencia de procesos de aprendizaje, por lo que los procesos vitales y los procesos de conocimiento son, en el fondo, la misma cosa [ambos procesos] descubren su lugar de encuentro, marcado desde siempre, en el centro de lo que constituye la vida, es decir, en el proceso de autoorganización tanto desde el plano biofísico, como desde el plano social. La vida quiere seguir siendo vida. Se gusta y se ama, y en consecuencia anhela ampliarse en más vida. (p.23)

Vale la pena recalcar, como lo concibe Assmann (2002), que la vida (y por tanto el aprendizaje) se da siempre en el placer, en el gozo, incluso en la ternura. Precisamente ese es el título de su libro Placer y Ternura en la Educación. Pero, ¿qué estamos haciendo? De modo que los y las jóvenes no se sienten partícipes de este mundo, pensamos que los conflictos se resuelven con violencia y con lucha.

Harari (2018) hace una crítica a la teoría de la educación, legado de la revolución industrial, como una cadena de producción.

En medio de la ciudad hay un gran edificio de hormigón dividido en muchas salas idénticas, cada una de ellas equipada con hileras de mesas y sillas. Al sonido de un timbre nos dirigimos a una de estas salas con otros treinta niños que nacieron el mismo año que nosotros. Cada hora entra un adulto en la sala y empieza a hablar. A todos ellos les paga el gobierno para eso. Uno de ellos nos habla de la forma de la Tierra, otro nos cuenta cosas del pasado de los humanos y un tercero nos explica aspectos del cuerpo humano. Es fácil reírse de este modelo, y casi todo el mundo está de acuerdo en que, con independencia de sus logros anteriores ahora se halla en crisis. Pero hasta ahora no hemos creado una alternativa viable. (p.292)

Hace algunos años, se crecía con conciencia de ciudadanía con cierta visión del mundo y de la vida que se quería vivir. Este tipo de conciencia sólo se generaba a partir de experiencias de aprendizaje que formaban personas como ciudadanos y ciudadanas planetarios en armonía con los suyos y con el medio que les rodeaba.

6. Desde pequeña estuve acompañada de la mano de mi madre, una mujer y gran historiadora, que aparte de historiadora fue una excelente narradora que a través de la tradición oral me contaba historias de mis antepasados, de mi pueblo y de mi país. ¡Qué mejor manera de aprender sobre historia que de la mano de nuestra propia familia! 
El aparato educativo actual forma un perfil de persona que aprende desde la obediencia, que se somete, que no es contestatario, que cree todo lo que se le enseña, que no hace preguntas, que se siente subyugado por un gobierno, por una economía, por la injustica y por el trabajo. Este tipo de ciudadano y ciudadana se muestra en un estado de conformismo de su realidad que ha sido explotado desde joven por el sistema hegemónico y que no siente que la sociedad le dé cabida para expresarse.

Gutiérrez (1990, p.30) decía que "el hombre inteligente no es el que mejor razona sino el que haciendo uso pleno y consciente de su intuición, se autorrealiza en el amor y el gozo de la vida”. Sin embargo, las personas en la sociedad actual desconocemos cómo autorrealizarnos, no sabemos expresar nuestras emociones, por lo que acudimos a manifestaciones de violencia. Al igual que la mariposa, el bebé desde que está en el útero aprende a través de las conexiones que la madre le provee con el exterior, es por esto que, estamos como seres humanos, habidos de relaciones más solidarias y sanas, que nos permitan aprender de los demás, aceptando legítimamente lo que el Otro tiene que decirnos.

\section{Referencias Bibliográficas}

Assman, H. (2002). Placer y Ternura en la Educación. Madrid, España: Narcea S.A.

Blanco, J., Seco, B., y Vargas, L. (2015). En busca del perfil del sujeto ciber-interlocutor para la mediación pedagógica en entornos virtuales. Revista REDpensar 4(1), 1-11.

Capra, F. (1992). Elpunto crucial. Buenos Aires, Argentina: Troquel.

Delors, J. (1994). La Educación encierra un tesoro. Informe a la UNESCO de la Comisión Internacional sobre la Educación para el Siglo XXI. Ciudad de México, México: Ediciones UNESCO. Recuperado de https:// bit.ly/2QBlGEA

Freire, P. (2008). Pedagogía del Oprimido. Madrid, España: Siglo XXI.

Grosfoguel, R. (2011). La descolonización del conocimiento: diálogo crítico entre la visión descolonial de Frantz Fanon y la sociología descolonial de Boaventura de Sousa Santos. Estados Unidos: Berkeley University.

Gutierrez, F. (2010). Las Nuevas Ciencias de la Vida. Polis, 223-233.

Gutiérrez, F. (1990). Siento, percibo, amo...Ergo sum. IDEAS, 1-39.

Gutiérrez, F., y Prieto, D. (2002). La Mediación Pedagógica. Apuntes para una educación a distancia alternativa. Madrid, España: L’Ullal Edicions

Harari, Y. (2018). 21 lecciones para el siglo XXI. Barcelona, España: Penguin Ramdon House .

Huxley, Jung, Watts, Maslow, Fromm, Castaneda, Aurobindo, Graves, Schumacher, Krihnamurti, KüblerRoss, Roszak, Rogers, Henderson, Capra, Bohm, Wilber, Sheldrake, Grof, Lovelock. (1994). Revista Integral. Especial Nueva Conciencia: Plenitud Personal y Equilibrio Planetario para el Siglo XXI. España: Integral.

Maturana, H. (2002). Transformación en la Convivencia. Providencia, Santiago, Chile: Dolmen Ediciones S.A.

Taylor, F. (1994). Paradigma de la Administración Cientifica. Buenos Aires, Argentina: El Ateneo.

Varela, F., Thompson, E., y Rosch, E. (1997). De Cuerpo Presente. Barcelona, España: Gedisa. 